\title{
Электронная структура четырехкомпонентных клатратных кристаллов системы $\mathrm{Ba}-\mathrm{Zn}-\mathrm{Si}-\mathrm{Ge}$
}

\author{
(C) Н.А. Борщ ${ }^{1}$, С.И. Курганский ${ }^{2}$ \\ ${ }^{1}$ Воронежский государственный технический университет, \\ 394026 Воронеж, Россия \\ ${ }^{2}$ Воронежский государственный университет, \\ 394036 Воронеж, Россия \\ E-mail: n.a.borshch@ya.ru
}

(Получена 19 апреля 2017 г. Принята к печати 31 августа 2017 г.)

Представлены результаты расчета электронной структуры четырехкомпонентных клатратных кристаллов на основе кремния и германия. Расчет проводился методом линеаризованных присоединенных плоских волн. Анализ результатов расчета позволил установить зависимость свойств кристаллов от соотношения числа атомов кремния и германия в элементарной ячейке, а также от положения замещающих атомов цинка.

DOI: $10.21883 /$ FTP.2018.03.45612.8615a

\section{1. Введение}

Синтез новых функциональных материалов всегда является актуальной задачей. Развитие современных технологий в различных сферах производства невозможно без применения качественно новых материалов. Наноклеточные кристаллы представляют собой идеальные объекты для конструирования новых функциональных материалов. Первый такой кристалл был синтезирован в 1965 году - это был силицид натрия с клатратной структурой [1], в которой внутри кремниевых сфероидов помещается атом натрия. Позднее были синтезированы кремниевые клатраты с инкапсулированными атомами калия, бария и других металлов [2-4]. Кроме того, был осуществлен синтез клатратов на основе германия и олова [5-7].

До начала 90-х годов прошлого века исследование клатратных кристаллов практически не проводилось. Однако, после того как в 90-х годах прошлого века Слэк выдвинул гипотезу [8], согласно которой соединения, в которых слабо связанные атомы могут колебаться в ограниченном объеме, должны обладать аномально низкой теплопроводностью при высокой электропроводности, клатраты оказались очевидными кандидатами на роль новых, перспективных термоэлектриков. Это послужило толчком к многочисленным работам по синтезу новых клатратов [9-12]. Оказалось, что существует сразу несколько возможностей влиять на свойства таких кристаллов. Прежде всего это сорт инкапсулированного атома. Показано как экспериментально, так и теоретически, что сорт этого атома влияет на электронные, сверхпроводящие, а также механические свойства кристалла $[2-4,13,14]$. Еще одна возможность - замещение атомов решетки-хозяина атомами другого сорта. Свойства кристалла при этом определяются как сортом замещающих атомов, так и их положением в элементарной ячейке [15-18].

В данной работе представлены результаты расчета электронной структуры четырехкомпонентных клатратных кристаллов на основе кремния и германия -
$\mathrm{Ba}_{8} \mathrm{Zn}_{8} \mathrm{Si}_{28} \mathrm{Ge}_{10}, \mathrm{Ba}_{8} \mathrm{Zn}_{8} \mathrm{Si}_{19} \mathrm{Ge}_{19}, \mathrm{Ba}_{8} \mathrm{Zn}_{8} \mathrm{Si}_{10} \mathrm{Ge}_{28}$. Анализ результатов расчета позволил установить зависимость свойств кристаллов от соотношения числа атомов кремния и германия в элементарной ячейке, а также от положения замещающих атомов цинка.

\section{2. Метод расчета}

Расчет электронной структуры клатратов проводился методом линеаризованных присоединенных плоских волн (ЛППВ) [19] в рамках локального приближения функционала плотности с использованием обменнокорреляционного потенциала в аппроксимации [20] и скалярно-релятивистского приближения [21]. В этом приближении учитываются все релятивистские эффекты, кроме спин-орбитального расщепления. Для расчетов электронной структуры использовался базис из 2517 ЛППВ. В разложении базисной функции по сферическим гармоникам учитывались вклады до $l_{\max }=7$.

Полученные в результате зонного расчета собственные функции $\psi_{i, \mathbf{k}}(\mathbf{r})$ и собственные значения энергии $E_{i}(\mathbf{k})$ использовались для расчета полных плотностей электронных состояний

$$
n(E)=\frac{2}{\Omega_{B Z}} \sum_{i} \int_{\Omega_{B Z}} \delta\left(E-E_{i}(\mathbf{k})\right) d \mathbf{k},
$$

локальных парциальных плотностей электронных состояний

$$
n_{s l}(E)=\frac{2}{\Omega_{B Z}} \sum_{i} \int_{\Omega_{B Z}} Q_{\mathbf{k}}^{s l} \delta\left(E-E_{i}(\mathbf{k})\right) d \mathbf{k}
$$

$\left(i\right.$ - номер энергетической зоны, $\Omega_{B Z}-$ объем первой зоны Бриллюэна, $Q_{\mathrm{k}}^{s l}$ определяет заряд $l$-типа симметрии, содержащийся внутри атомной сферы, окружающей в элементарной ячейке атомы $s$-типа).

Твердые растворы системы $\mathrm{Ba}-\mathrm{Zn}-\mathrm{Si}-\mathrm{Ge}$ кристаллизуются в примитивную кубическую ячейку пространственной группы Pm3n [22]. На одну элементарную ячейку приходится два полиэдра $\mathrm{Si}(\mathrm{Ge})_{20}$ и шесть полиэдров 

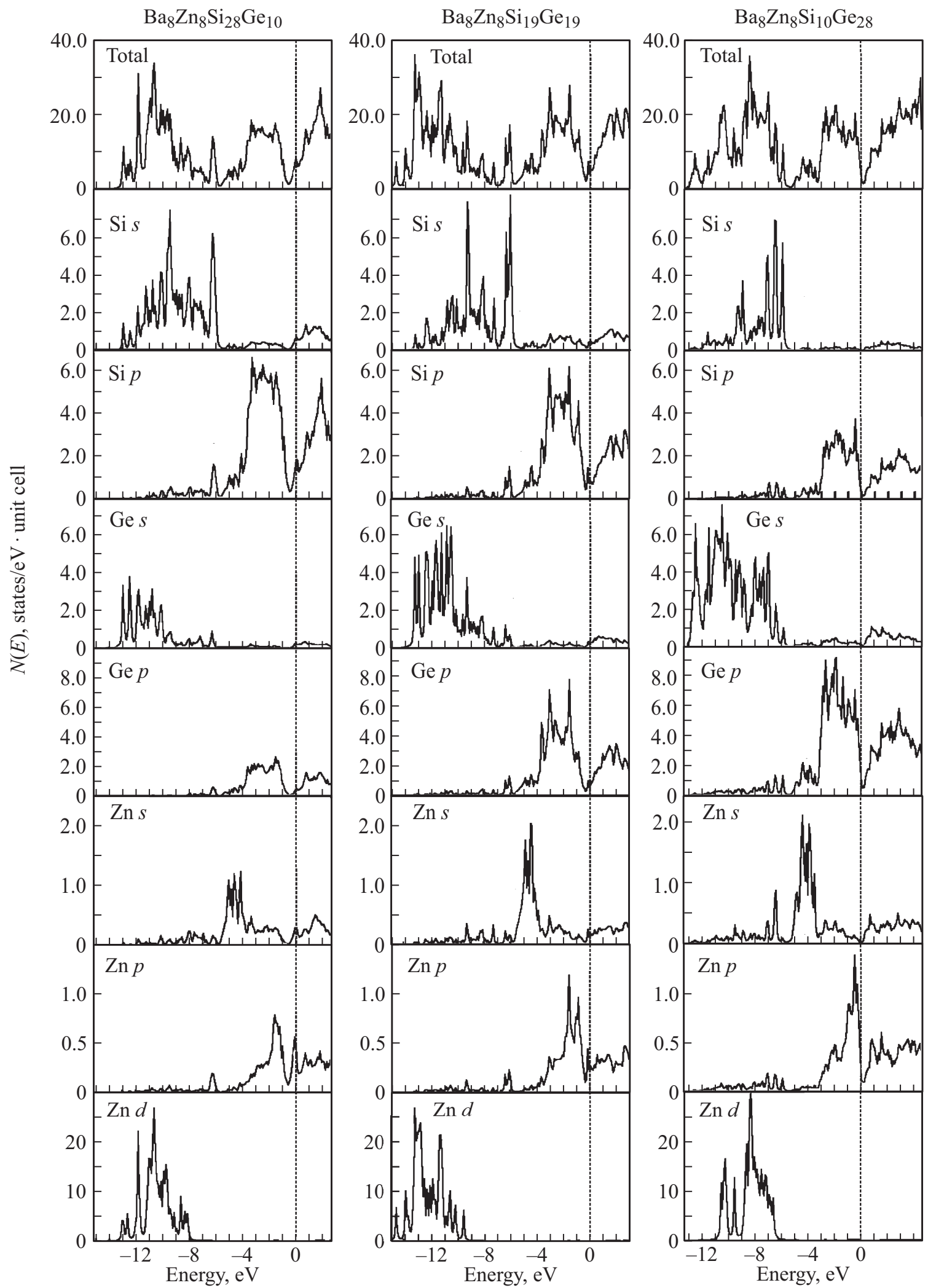

Рис. 1. Полные и парциальные плотности электронных состояний в клатратах системы $\mathrm{Ba}-\mathrm{Zn}-\mathrm{Si}-\mathrm{Ge}$.

$\mathrm{Si}(\mathrm{Ge})_{24}$. Атомы бария заполняют пустоты в полиэдрах. В элементарной ячейке имеется две неэквивалентные кристаллографические позиции атомов бария $-2 a$ и $6 b$ и три неэквивалентных позиции атомов клатратной решетки - $6 c, 16 i$ и $24 k$ (рис. 1) [22]. Шесть атомов цинка полностью замещают атомы клатратной подрешетки в 

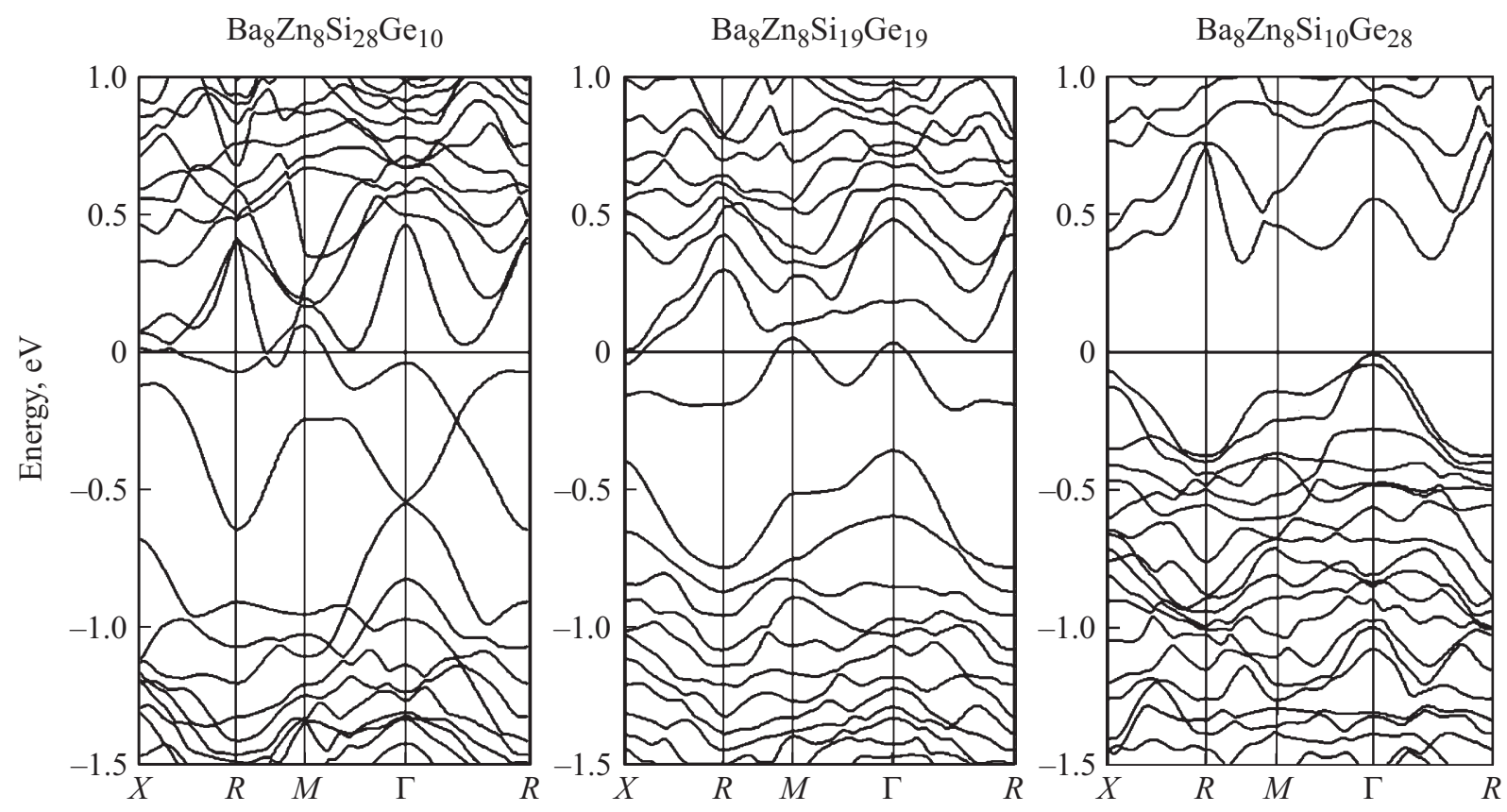

Рис. 2. Структура энергетических зон в клатратах системы $\mathrm{Ba}-\mathrm{Zn}-\mathrm{Si}-\mathrm{Ge}$ около уровня Ферми.

позиции $6 c$, а еще два атома цинка - в позициях $16 i$ и(или) $24 k$. В клатрате $\mathrm{Ba}_{8} \mathrm{Zn}_{8} \mathrm{Si}_{28} \mathrm{Ge}_{10}$ замещение происходит по схеме $6 c+24 k$, в клатрате $\mathrm{Ba}_{8} \mathrm{Zn}_{8} \mathrm{Si}_{19} \mathrm{Ge}_{19}-$ $6 c+16 i+24 k$, а в клатрате $\mathrm{Ba}_{8} \mathrm{Zn}_{8} \mathrm{Si}_{10} \mathrm{Ge}_{28}-6 c+16 i$.

\section{3. Зонная структура и плотность состояний}

Зонную картину валентной зоны всех клатратов образуют 132 энергетические зоны. Как показал расчет парциальных плотностей электронных состояний (рис. 1), в низкоэнергетической части валентной зоны доминирует вклад валентных $\mathrm{Si}(\mathrm{Ge}) s$-состояний, в высокоэнергетической части - вклад валентных $\mathrm{Si}(\mathrm{Ge})$-состояний. Парциальные плотности $\mathrm{Si} s$ - и $\mathrm{Ge} s$-состояний становятся тем более локализованными, чем меньше содержание соответствующего элемента в элементарной ячейке клатрата. При этом $s$-состояния германия локализуются у дна валентной зоны, а $s$-состояния кремния - на несколько эВ выше. p-состояния кремния и германия расположены в одной энергетической области у вершины валентной зоны. Zn $3 d$-состояния локализованы у дна валентной зоны, а $\mathrm{Zn} 4 s$ - и $\mathrm{Zn} 4 p$-состояния вносят заметный вклад в электронный спектр высокоэнергетической части валентной зоны.

Атомы решетки-хозяина стремятся к образованию тетраэдрических связей. Для этого необходимо, чтобы замещающие атомы Zn имели четырехэлектронную валентную конфигурацию. Атомы Ва являются донорами электронов для клатратной решетки. Каждый атом бария отдает решетке-хозяину по два валентных $6 s$-электрона. Таким образом, от восьми атомов бария клатратная решетка-хозяин получает 16 дополнительных электронов на элементарную ячейку. В рассматриваемых клатратах для образования связей $\mathrm{Zn}-\mathrm{Si}(\mathrm{Ge})$ нужно 16 электронов, поэтому все электроны, отданные атомами Ва, используются для этого, и в ячейке нет ни дефицита, ни избытка электронов.

На рис. 2 показана структура энергетических зон в прифермиевской области клатратов. В клатрате $\mathrm{Ba}_{8} \mathrm{Zn}_{8} \mathrm{Si}_{28} \mathrm{Ge}_{10}$ от основной части валентной зоны отщепляются две энергетические зоны. Одна из них пересекает уровень Ферми. В результате уровень Ферми пересекают две энергетические зоны - верхняя зона валентной полосы и нижняя зона зоны проводимости, которые перекрываются по энергетической шкале, что характерно для полуметаллов или „плохих“ металлов. $\mathrm{B}$ клатрате $\mathrm{Ba}_{8} \mathrm{Zn}_{8} \mathrm{Si}_{19} \mathrm{Ge}_{19}$ от основной части валентной зоны отщепляется только одна энергетическая зона, которая также пересекает уровень Ферми, перекрываясь по энергетической шкале с частично заполненной нижней зоной зоны проводимости. Это значит, что клатрат $\mathrm{Ba}_{8} \mathrm{Zn}_{8} \mathrm{Si}_{19} \mathrm{Ge}_{19}$ является типичным полуметаллом. В клатрате $\mathrm{Ba}_{8} \mathrm{Zn}_{8} \mathrm{Si}_{10} \mathrm{Ge}_{28}$ нет отщепленных зон, верхняя энергетическая зона валентной полосы полностью заполнена и не пересекает уровень Ферми, что свидетельствует о том, что клатрат $\mathrm{Ba}_{8} \mathrm{Zn}_{8} \mathrm{Si}_{10} \mathrm{Ge}_{28}$ является непрямозонным полупроводником с шириной запрещенной зоны 0.29 эВ.

Наличие или отсутствие отщепленных зон у потолка валентной зоны клатрата объясняется особенностями расположения замещающих атомов цинка в его элементарной ячейке. Как упоминалось выше, шесть атомов Zn занимают в элементарной ячейке клатрата $6 c$-позицию, а два - 16i- и(или) 24k-позицию в зависимости от соотношения числа атомов $\mathrm{Si} / \mathrm{Ge}$. Ближайшее окружение 


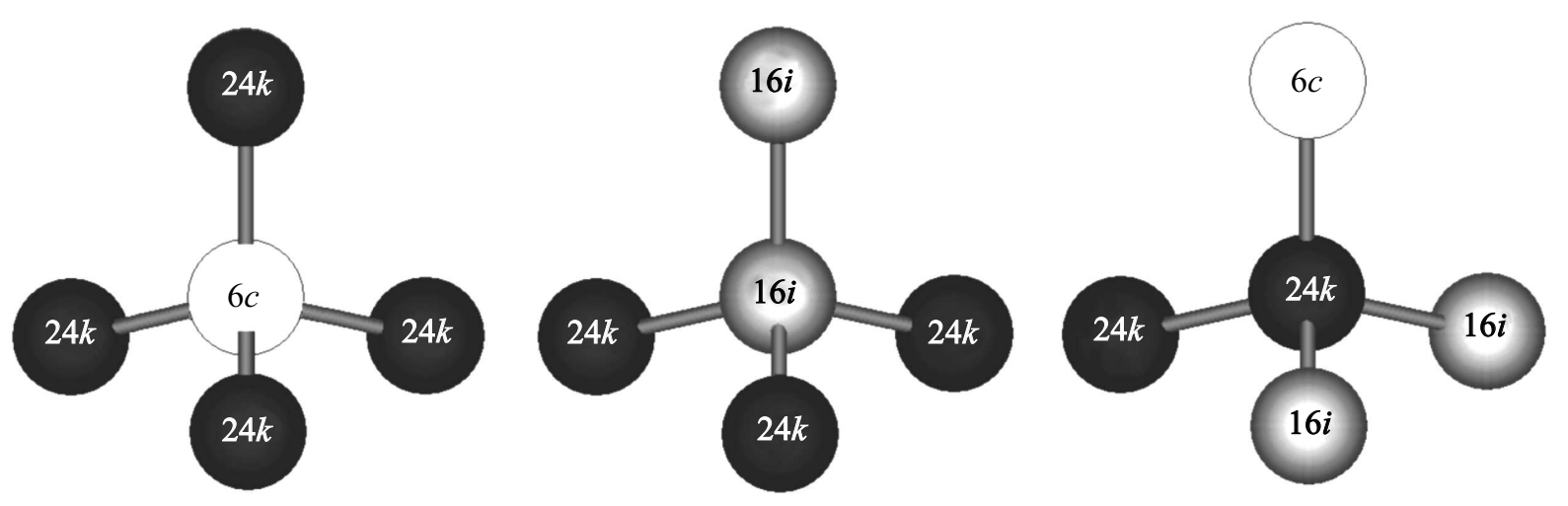

Рис. 3. Координационные тетраэдры атомов клатратной решетки из неэквивалентных кристаллографических позиций.
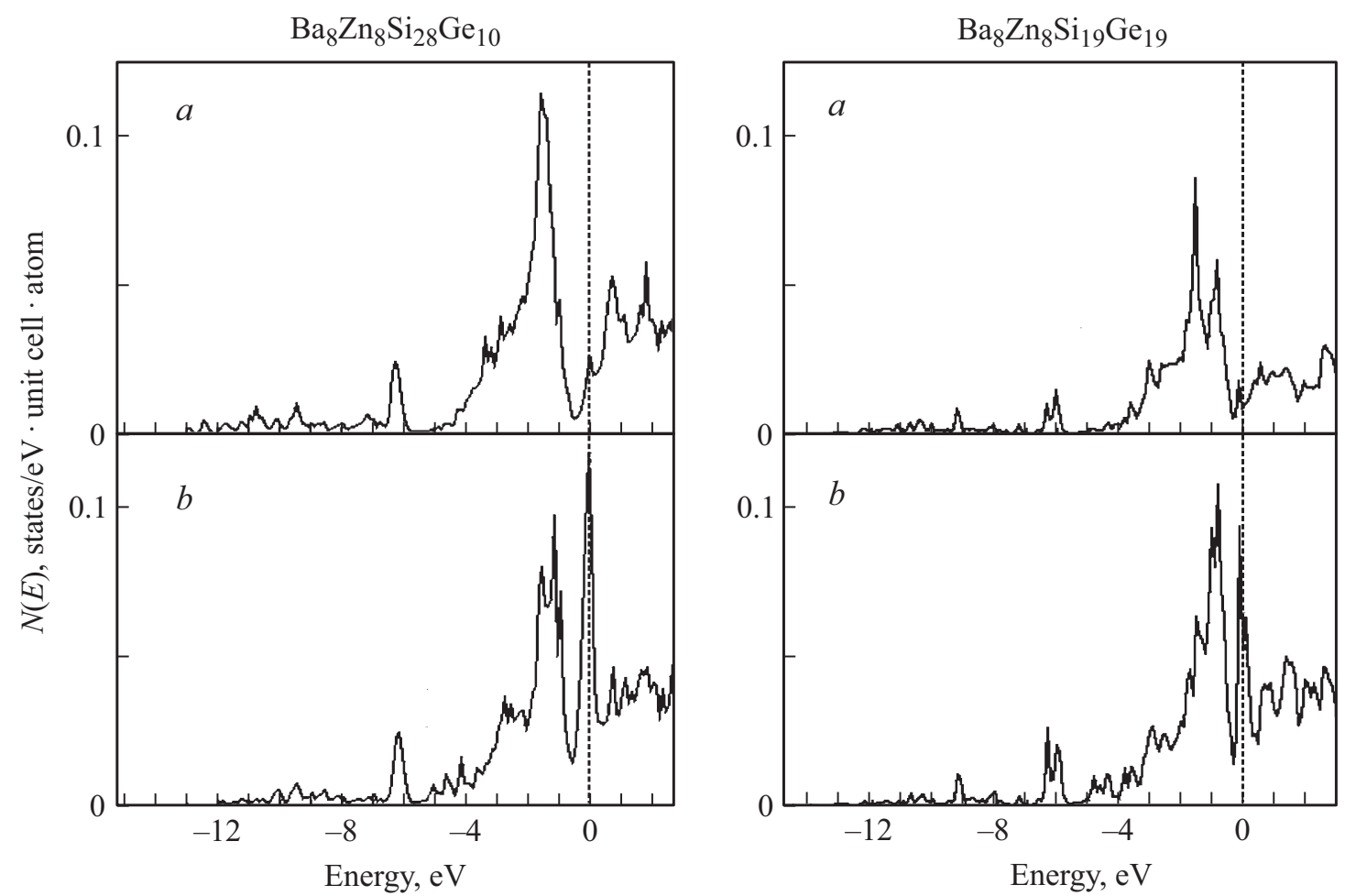

Рис. 4. Локальные парциальные плотности электронных состояний атомов цинка из разных кристаллографических позиций в клатратах системы $\mathrm{Ba}-\mathrm{Zn}-\mathrm{Si}-\mathrm{Ge}$.

$6 c$-атома состоит из трех $24 k$-атомов (рис. 3 ). При этом в силу симметрии элементарной ячейки в окружении $6 c$-атома может быть не более одного замещающего атома цинка. Следовательно, в клатратах с $6 c+24 k$ замещениями обязательно есть пара соседствующих атомов цинка ( $6 c$-атом и $24 k$-атом), и число этих пар равно числу замещающих атомов в $24 k$-позиции.

Кристаллографическая позиция атомов цинка влияет на особенности локальных парциальных плотностей электронных состояний. Как показал расчет, плотность $4 p$-состояний атомов цинка, имеющих в своем окружении атомы цинка, расщепляется около уровня Ферми (рис. 4). Плотность $4 p$-состояний таких атомов имеет два основных максимума - максимум при энергии
-1.5 эВ, соответствующий связям $\mathrm{Zn}-\mathrm{Si}(\mathrm{Ge})$, и максимум непосредственно около уровня Ферми, который соответствует менее прочным связям $\mathrm{Zn}-\mathrm{Zn}$. В элементарной ячейке клатрата $\mathrm{Ba}_{8} \mathrm{Zn}_{8} \mathrm{Si}_{28} \mathrm{Ge}_{10}$ с $6 c+24 k$ замещениями имеются две связи $\mathrm{Zn}-\mathrm{Zn}$, которым соответствуют две отщепленные энергетические зоны. В клатрате $\mathrm{Ba}_{8} \mathrm{Zn}_{8} \mathrm{Si}_{19} \mathrm{Ge}_{19}$ имеется только два соседствующих атома цинка, т.е. одна связь $\mathrm{Zn}-\mathrm{Zn}$. Этой связи соответствует одна отщепленная энергетическая зона. В клатрате $\mathrm{Ba}_{8} \mathrm{Zn}_{8} \mathrm{Si}_{10} \mathrm{Ge}_{28}$ с $6 c+16 i$ замещениями в ближайшем окружении $16 i$-атомов имеются три $24 k$-атома и один $16 i$-атом (рис. 3 ), следовательно, все замещающие атомы цинка окружены только атомами кремния. Поэтому в плотности $\mathrm{Zn} 4 p$-состояний в этом 
клатрате отсутствует максимум на уровне Ферми, а значит, и отщепленные зоны у потолка валентной зоны (см. рис. 2).

Таким образом, можно заключить, что в клатратах ряда $\mathrm{Ba}-\mathrm{Zn}-\mathrm{Si}-\mathrm{Ge}$ кристаллографическая позиция замещающих атомов цинка определяет характер проводимости кристалла и ширину энергетической щели между валентной зоной и зоной проводимости клатрата.

\section{4. Заключение}

Зонная картина в наноклеточных твердых растворах системы $\mathrm{Ba}-\mathrm{Zn}-\mathrm{Si}-\mathrm{Ge}$ имеет особенности, общие для всех рассмотренных кристаллов. У дна валентной зоны наряду с $3 d$-состояниями атомов цинка доминирует вклад валентных $s$-состояний германия и кремния, в высокоэнергетической части валентной зоны основной вклад вносят $\mathrm{Si} 3 p$ - и $\mathrm{Ge} 4 p$-состояния. Плотность электронных состояний около уровня Ферми формируют состояния атомов цинка и тех атомов кремния и германия, которые имеют в ближайшем окружении атомы цинка.

Характер проводимости и ширина энергетической щели между валентной зоной и зоной проводимости в твердых растворах $\mathrm{Ba}-\mathrm{Zn}-\mathrm{Si}-\mathrm{Ge}$ зависит от кристаллографической позиции атомов цинка, которая в свою очередь определяется соотношением числа атомов кремния и германия в элементарной ячейке кристалла. Наличие атомов цинка в $24 k$-позиции приводит к возникновению эффекта отщепленных энергетических зон, вследствие которого ширина запрещенной зоны уменьшается. Клатраты $\mathrm{Ba}_{8} \mathrm{Zn}_{8} \mathrm{Si}_{28} \mathrm{Ge}_{10}$ и $\mathrm{Ba}_{8} \mathrm{Zn}_{8} \mathrm{Si}_{19} \mathrm{Ge}_{19}$ являются полуметаллами, а клатрат $\mathrm{Ba}_{8} \mathrm{Zn}_{8} \mathrm{Si}_{10} \mathrm{Ge}_{28}-$ непрямозонным полупроводником с шириной запрещенной зоны 0.29 эВ.

\section{Список литературы}

[1] J.S. Kasper, P. Hagenmuller, M. Pouchard, C. Cros. Science, 150, 1713 (1965).

[2] J.S. Tse, S. Desgreniers, Zhi-qiang Li, M.R. Ferguson, Y. Kawazoe. Phys. Rev. B, 89, 195507 (2002).

[3] H. Fukuoka, J. Kiyoto, S. Yamanaka. Inorg. Chem., 42, 2933 (2003).

[4] F. Shimizu, Y. Maniwa, K. Kume, K. Kawaji, S. Yamanaka, M. Ishikawa. Phys. Rev. B, 54, 13242 (1996).

[5] N. Melnychenko-Koblyuk, A. Grytsiv, L. Fornasari. J. Phys.: Condens. Matter, 19, 046203 (2007).

[6] H. Fukuoka, S. Yamanaka, K. Yoza, L. Häming. J. Solid State Chem., 151, 117 (2000).

[7] S. Bobev, S.C. Sevov. J. Solid State Chem., 153, 92 (2000).

[8] G.A. Slack, Mater. Res. Symp. Proc., 478, 47 (1997).

[9] B.B. Iversen, E Anders, E.C. Palmqvist, D.E. Cox, A. George, S. Nolas, B. Galen, D. Stucky, N.P. Blake, H. Meti. J. Solid State Chem., 149, 455 (2000).

[10] Ya. Mudryk, P. Rogl, C. Paul, S. Berger, E. Bauer, G. Hilscher, C. Godart, H. Noël. J. Phys.: Condens. Matter, 14, 7991 (2002).
[11] H.F. Wang, K.F. Cai, H. Li, D.H. Yu, X. Wang, C.W. Zhou, X.L. Li, Y.Y. Wang, B.J. An, Y. Du. J. Alloys Comp., 491, 684 (2010).

[12] M. Falmbigl, N. Nasir, A. Grytsiv, P. Rogl, S. Seichter, A. Zavarsky, E. Royanian, E. Bauer. J. Phys. D: Appl. Phys., 45, 215308 (2012).

[13] A. Bentien, E. Nishibori, S. Paschen, B.B. Iversen. Phys. Rev. B, 71, 144107 (2005).

[14] S. Paschen, W. Carrillo-Cabrera, A. Bentien, V.H. Tran, M. Baenitz, Yu. Grin, F. Steglich. Phys. Rev. B, 64, 214404 (2001).

[15] H. Fukuoka, J. Kiyoto, S. Yamanaka. J. Solid State Chem., 175, 237 (2003).

[16] Н.А. Борщ, Н.С. Переславцева, С.И. Курганский. ФТП, 45 (6), 729 (2011).

[17] N. Jaussaud, P. Gravereau, S. Pechev, B. Chevalier. C. R. Chimie, 8, 39 (2005).

[18] Yang Li, Yang Liua, Ning Chen, Guohui Cao, Zhaosheng Feng, J.H. Ross. J. Phys. Lett. A, 345, 398 (2005).

[19] S.N. Vosko, L. Wilk, M. Nusair. Can. J. Phys., 58, 1200 (1980).

[20] D.D. Koelling, G.O. Arbman. J. Phys. F, 5, 2041 (1975).

[21] A.H. MacDonald, W.E. Pickett, D.D. Koelling. J. Phys. C, 13, 2675 (1980).

[22] N. Nasir, A. Grytsiv, N. Melnychenko-Koblyuk, P. Rogl, I. Bednar, E. Bauer. J. Solid State Chem., 183, 2329 (2010).

Редактор Г.А. Оганесян

\section{Electronic structure of four-element clathrates $\mathrm{Ba}-\mathrm{Zn}-\mathrm{Si}-\mathrm{Ge}$}

\author{
N.A. Borshch ${ }^{1}$, S.I. Kurganskii ${ }^{2}$ \\ ${ }^{1}$ Voronezh State Technical University, \\ 394026 Voronezh, Russia \\ 2 Voronezh State University, \\ 394036 Voronezh, Russia
}

\begin{abstract}
Theoretical investigation of electronic structure and spectral characteristics of four-element clathrates $\mathrm{Ba}-\mathrm{Zn}-\mathrm{Si}-\mathrm{Ge}$ are presented. Band structure, total and partial density of states were obtained as results of the calculation. Influence of the sort, crystallographic position and numer of substituted $\mathrm{Zn}$ atoms on a electron spectra was considered. In calculation the linearized augmented plane wave method was used.
\end{abstract}

